\title{
Time-energy correlations in solar flare occurrence
}

\author{
E. Lippiello ${ }^{1}$, L. de Arcangelis ${ }^{2,3}$, and C. Godano ${ }^{1}$ \\ 1 Department of Environmental Sciences and CNISM, Second University of Naples, 81100 Caserta, Italy \\ e-mail: lippiello@sa.infn.it \\ 2 Department of Information Engineering and CNISM, Second University of Naples, 81031 Aversa (CE), Italy \\ 3 Institute Computational Physics for Engineering Materials, ETH, Zürich, Switzerland
}

Received 1 December 2009 / Accepted 5 February 2010

\section{ABSTRACT}

\begin{abstract}
Context. The existence of time-energy correlations in flare occurrence is still an open and much debated problem. Aims. This study addresses the question whether statistically significant correlations are present between energies of successive flares as well as energies and waiting times.

Methods. We analyze the GOES catalog with a statistical approach based on the comparison of the real catalog with a reshuffled one where energies are decorrelated. This analysis reduces the effect of background activity and is able to reveal the role of obscuration. Results. We show the existence of non-trivial correlations between waiting times and energies, as well as between energies of subsequent flares. More precisely, we find that flares close in time tend to have the second event with large energy. Moreover, after large flares the flaring rate significantly increases, together with the probability of other large flares.

Conclusions. Results suggest that correlations between energies and waiting times are a physical property and not an effect of obscuration. These findings could give important information on the mechanisms for energy storage and release in the solar corona.
\end{abstract}

Key words. Sun: flares - methods: data analysis

\section{Introduction}

Solar flares are violent explosions of magnetic energy in the solar corona. The theory of magnetic reconnection (see Priest \& Forbes 2002, for a review) represents the most plausible and widely accepted explanation for flare occurrence, although a definite and clear explanation of the mechanisms at the basis of flare triggering is still lacking. A well-established property of flare occurrence is the power law decay of the peak-flux energy distribution (Lee et al. 1993; Aschwanden et al. 1998; Crosby et al. 1998), a property shared by other stochastic physical phenomena like earthquakes (de Arcangelis et al. 2006). Several mechanisms have been proposed to reproduce the above experimental findings. In the Rosner and Vaiana (RV) model (Rosner \& Vaiana 1978) it is assumed that flare occurrence is an uncorrelated Poisson process where the energy grows at a rate proportional to the internal energy of the system. Given this assumption, the energy storage follows an exponential temporal growth, interrupted at random when the system releases all the stored energy in a flare. This mechanism accounts for the power law in the size distribution and predicts correlations between the storage time and the released energy. More precisely, the later a flare will occur the higher the energy will be. Also the avalanche model (Lu \& Hamilton 1991; Hamon et al. 2002), which describes solar flares as energy relaxation events in a system driven at a constant rate, correctly predicts scale-free behavior for the peak-flux energy distribution. Within this approach, flare occurrence is again a Poisson process, as in the RV model, but occurrence times and energies are uncorrelated like in standard self-organized models (Jensen 1998). Finally, power law behavior for the size distribution is also consistent with models assuming correlations between the waiting times $\Delta t$ of successive bursts, as in a shell model of magneto-hydrodynamic turbulence (Boffetta et al. 1999). This model reproduces the experimental power law decay of the waiting time distribution $P(\Delta t)$ which can also be obtained however by means of an uncorrelated piecewise Poisson process (Wheatland et al. 2002; Norman et al. 2001).

The investigation of correlations between flare energies and waiting times is a useful tool to distinguish among different triggering mechanisms. This problem has been addressed in a series of papers (Crosby et al. 1998; Wheatland et al. 1998; Wheatland 2000), providing no clear evidence for time-energy correlations. In particular, Wheatland et al. (1998) detected small correlations at short time and interpreted them as a spurious effect of obscuration, i.e. long-lasting events may hide subsequent small events. By properly taking into account obscuration effects, Wheatland concluded that no significant correlations are present. In this paper we present a novel analysis of experimental catalogs providing evidence for time-energy correlations in flare occurrence.

\section{Data analysis and methods}

In our analysis we used data from the GOES (Geostationary Operational Environmental Satellite) catalog (ftp://ftp. ngdc.noaa.gov/STP/SOLAR_DATA/SOLAR_FLARES) for the solar cycles $21-23$ in the years 1977 to 2007 . We include only C1.4 class flares in the analysis (peak flux energy larger than $E_{0}=1.4 \times 10^{-6} \mathrm{Wm}^{-2}$ ). Each flare in the catalog is characterized by its starting time $t_{i}$ and its peak-flux energy $E_{i}$, in the following called "energy" to simplify notation and measured in unit of $\mathrm{Wm}^{-2}$, counting 38575 flares with $E \geq E_{0}$. In order to enhance the eventual presence of temporal and energy correlations among flares, we performed a recently proposed (Lippiello et al. 2008a) statistical analysis of the catalog. We computed the probability to have a given quantity $x_{i}$ greater than $X$ conditioned to $\Delta t_{i}$ being smaller than $T, P\left(x_{i}>X \mid \Delta t_{i}<T\right)$. Here, 
$\Delta t_{i}=t_{i}-t_{i-1}$ is the waiting time and the quantity $x_{i}$ represents, depending on cases, the energy of the $i$ th flare $x_{i}=E_{i}$, the energy of the previous flare $x_{i}=E_{i-1}$ or the the energy ratio between successive flares, $x_{i}=E_{i} / E_{i-1}$. The quantity $P\left(x_{i}>X \mid \Delta t_{i}<T\right)$ is given by $P\left(x_{i}>X \mid \Delta t_{i}<T\right) \equiv \frac{N(X, T)}{N(T)}$, where $N(X, T)$ is the number of couples of subsequent events fulfilling both $x_{i}>X$ and $\Delta t_{i}<T$, whereas $N(T)$ is the number of couples satisfying only the condition $\Delta t_{i}<T$. In order to detect eventual correlations in the catalog, we introduced the probability difference $\delta P\left(x_{i}>X \mid \Delta t_{i}<T\right) \equiv P\left(x_{i}>X \mid \Delta t_{i}<T\right)-P\left(x_{i}>X\right)$, where $P\left(x_{i}>X\right)$ is the unconditional probability to have $x_{i}>X$. A positive (negative) $\delta P$ indicates a higher (lower) probability to find flares with $x_{i}>X$ if one restricts the analysis to couples with $\Delta t_{i}>T$. Hence, values of $\delta P$ different from zero suggest the existence of correlations between $x_{i}$ and $\Delta t_{i}$. Evidently, because of statistical fluctuations, $\delta P$ is never exactly equal to zero even in catalogs where $x_{i}$ and $\Delta t_{i}$ are uncorrelated. In order to explicitly take into account the role of statistical fluctuations, we computed the quantity $P^{*}\left(x_{i}>X \mid \Delta t_{i}<T\right)$, defined as $P$, but in a cata$\log$ where flare energies were randomly reshuffled. The comparison with thereshuffled catalog is the basis of the surrogate data technique (Theiller et al. 1992; Schreiber \& Schmitz 2000), recently applied to investigate coherent structures in space plasma (Sahraoui 2008). In the reshuffled catalog $x_{i}$ was by construction uncorrelated to $\Delta t_{i}$ and $P^{*}\left(x_{i}>X \mid \Delta t_{i}<T\right)$ fluctuated around its average value $P\left(x_{i}>X\right)$. The amplitude of these fluctuations defined the significance level, $\sigma(X, T)$, which allowed us to distiguish between the presence and absence of correlations. The method is schematically presented in Fig. 1 for $x_{i}=E_{i}$, $X=E=3 E_{0}$ and $T=1 \mathrm{~h} . P^{*}\left(E_{i}>E \mid \Delta t_{i}<T\right)$ takes different values for each realization of the reshuffled catalog. We produced $10^{5}$ independent realizations of the catalog with reshuffled energies and observed that $P^{*}\left(E_{i}>E \mid \Delta t_{i}<T\right)$ is Gaussian-distributed with a mean $P\left(E_{i}>E\right)$ and a standard deviation $\sigma(E, T)$. Similar results were obtained for other values of $E$ and $T$ and for the other definitions of $x_{i}$. Therefore, $\left|\delta P\left(x_{i}>X \mid \Delta t_{i}<T\right)\right|>\sigma(X, T)$ indicates that energies in the real catalog follow a significantly different organization than events in the reshuffled catalog.

\section{Results}

In Fig. 2a we plot $\delta P\left(E_{i}>E \mid \Delta t_{i}<T\right)$ and in Fig. 2b $\delta P\left(E_{i-1}>\right.$ $\left.E \mid \Delta t_{i}<T\right)$ for different values of $T$. The standard deviation for each data point $\sigma(E, T)$ is represented as the error bar. In Fig. 2a we notice that $\delta P\left(E_{i}>E \mid \Delta t_{i}<T\right)$ takes always positive values beyond error bars. This implies that in the real catalog the number of couples fulfilling both conditions is greater than in catalogs where energies and intertimes are uncorrelated. More precisely we find that for each given value of $E, \delta P\left(E_{i}>E \mid \Delta t_{i}<T\right)$ decreases by increasing $T$. This implies that the probability to find flare couples with the second flare energy higher than $E$ decreases if one includes events with larger $\Delta t$ in the analysis. This result is disagrees with what is expected according to the RV model, which predicts larger flares after longer waiting times. On the other hand, obscuration can represent a possible explanation of the above result. Indeed, according to the selection procedure, for flares close in time, the second event is recorded in the catalog only if it produces an increase in the flux of at least $40 \%$ of the level of the previous flare. Moreover, the light curve of large flares may hide smaller flares close in time. Obscuration effects then reduce the probability to find a small

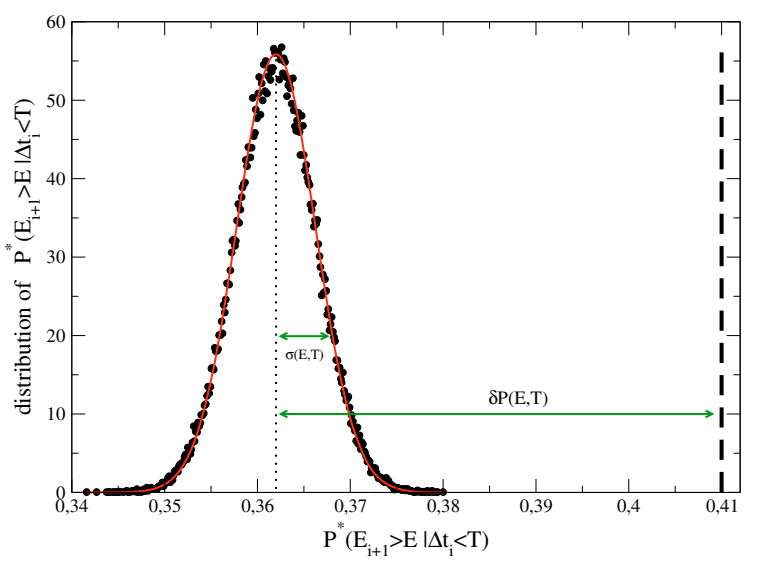

Fig. 1. Distribution of $P^{*}\left(E_{i}>E \mid \Delta t_{i}<T\right)$ for $E=3 E_{0}, T=1 \mathrm{~h}$ and $10^{5}$ realizations of the reshuffled catalog represented by black circles. The continuous red line is the fit with a Gaussian distribution with an average value of 0.3619 (dotted line) and a standard deviation $\sigma(E, T)=$ $6.5 \times 10^{-3}$. The dashed line indicates the value $P\left(E_{i}>E \mid \Delta t_{i}<T\right)=$ 0.4098 obtained in the real catalog.

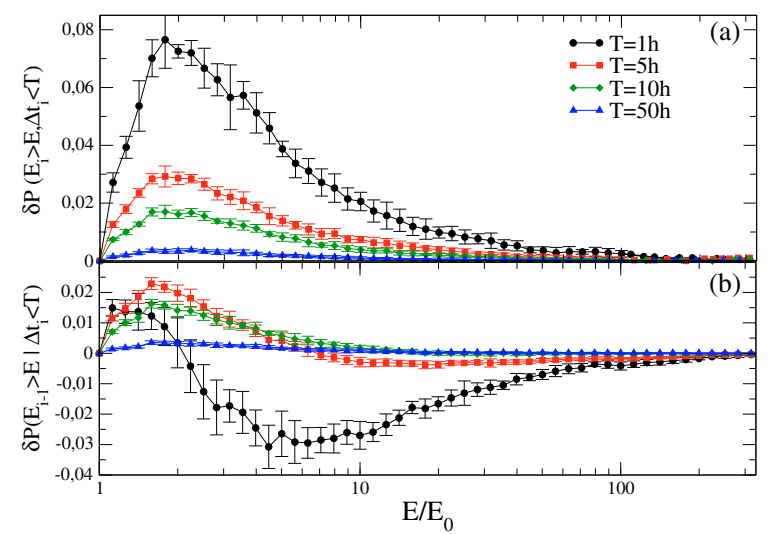

Fig. 2. Probability difference $\delta P\left(E_{i}>E \mid \Delta t_{i}<T\right)$ a) and $\delta P\left(E_{i-1}>\right.$ $\left.\left.E \mid \Delta t_{i}<T\right) \mathbf{b}\right)$ as a function of $E / E_{0}$ for different values of $T$. For each data point the error bar is the standard deviation $\sigma(E, T)$.

flare after a short waiting time and may introduce spurious correlations between waiting time and the successive flare energy.

We then explicitly explored the role of obscuration on $\delta P\left(E_{i}>E \mid \Delta t_{i}<T\right)$. As a first analysis we considered only flare couples with a temporal distance greater than $k$ times the duration of the first flare. Spurious effects due to obscuration should disappear by increasing $k$. We observed (Fig. 3a) that this extra condition did not reduce correlations between waiting times and energies, since they are still present also for the highest values of $k$. To further prove that correlations cannot be attributed to spurious effects related to obscuration, we performed the same analysis as in Fig. 1a, setting different lower energy thresholds $e_{\mathrm{th}}$. Indeed, by increasing the value of the minimum energy required for flares to be included in the analysis, the percentage of flares hidden by obscuration was reduced and obscuration effects should progressively decrease. In Fig. 3 b we plot the results for different energy thresholds $e_{\text {th }}$ as a function of $E / e_{\mathrm{th}}$. No significant dependence on $e_{\mathrm{th}}$ is detected, indicating that correlations cannot be attributed to obscuration effects. We conclude that for couples of events close in time it is more probable to have the second event with a high energy than for events distant in time.

In Fig. $2 b$ we performed the same analysis as in Fig. 2a for the correlations between the waiting time and the previous flare 
E. Lippiello et al.: Time-energy correlations in solar flare occurrence
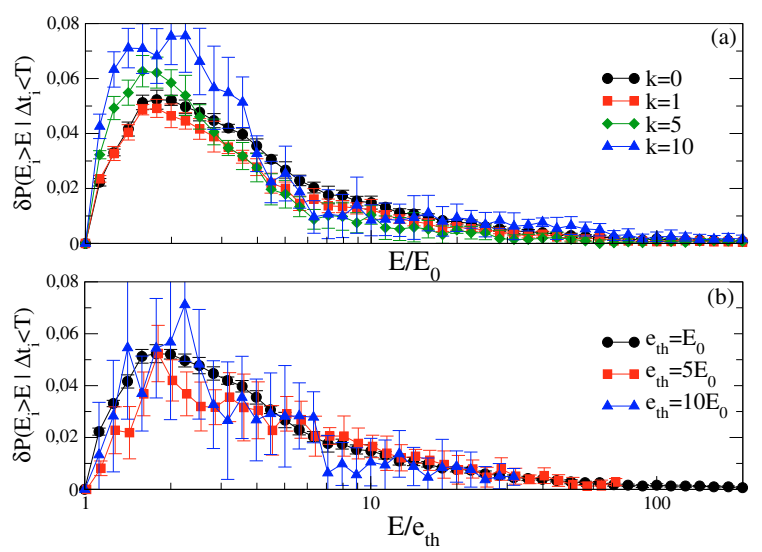

Fig. 3. a) Probability difference $\delta P\left(E_{i-1}>E \mid \Delta t_{i}<T\right)$ as a function of $E / E_{0}$ for $T=1 \mathrm{~h}$ and different $k$ values. b) Same quantity as function of $E / e_{\mathrm{th}}$ for $T=1 \mathrm{~h}$ and different values of $e_{\mathrm{th}}$. For each data point the error bar is the standard deviation $\sigma(E, T)$.

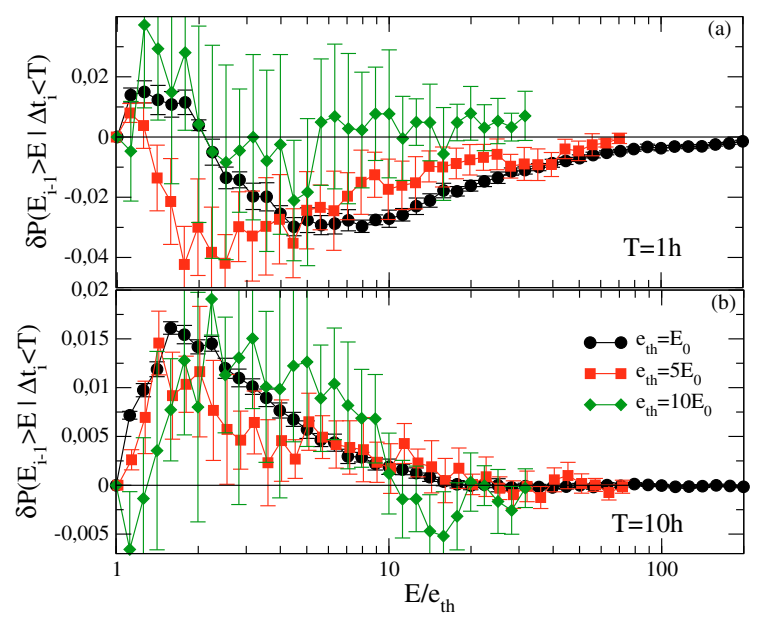

Fig. 4. Probability difference $\delta P\left(E_{i-1}>E \mid \Delta t_{i}<T\right)$ as a function of $E / e_{\mathrm{th}}$ for $\left.T=1 \mathrm{~h} \mathbf{a}\right)$ and $T=10 \mathrm{~h} \mathrm{~b}$ ) for different values of $e_{\mathrm{th}}$. For each data point the error bar is the standard deviation $\sigma(E, T)$.

energy. In contrast to Fig. 2a the quantity $\delta P\left(E_{i-1}>E \mid \Delta t_{i}<T\right)$ is not always positive now. In particular for $T=1 \mathrm{~h}, \delta P\left(E_{i-1}>\right.$ $\left.E \mid \Delta t_{i}<T\right)$ is positive at small $E$ and negative at large $E$. In order to verify if the behavior at small $T$ is affected by obscuration, we performed the same analysis as in Fig. 3b, imposing the condition on the lower energy threshold $e_{\text {th }}$. In Fig. 4a we present $\delta P\left(E_{i-1}>E \mid \Delta t_{i}<T\right)$ for $T=1 \mathrm{~h}$ and different energy thresholds. We found that data strongly depend on $e_{\mathrm{th}}$, in particular for $e_{\mathrm{th}}=10 E_{0}, \delta P\left(E_{i-1}>E \mid \Delta t_{i}<T\right)$ is comparable with the statistical fluctuations. This analysis suggests that the correlations observed at small $\Delta t$ are related to obscuration effects. Conversely, data for $T=10 \mathrm{~h}$ are not significantly affected by $e_{\text {th }}$ and $\delta P\left(E_{i-1}>E \mid \Delta t_{i}<T\right)$ always takes positive values beyond error bars (Fig. 4b). This result indicates that correlations detected for events with temporal distances between $1 \mathrm{~h}$ and $10 \mathrm{~h}$ are not significantly affected by obscuration. We conclude that couples of events distant in time between $1 \mathrm{~h}$ and $10 \mathrm{~h}$ tend to have the first event with a higher energy than the one expected for a process where time and energy are uncorellated.

To obtain more detailed information on correlations between $E_{i-1}$ and $\Delta t$, we considered the quantity $d p\left(E, \alpha_{1}, \alpha_{2}\right) \equiv$ $\delta P\left(E_{i-1}=E \mid \alpha_{1}<\Delta t_{i}<\alpha_{2}\right)$, i.e. the difference between conditional and unconditional probability densities to have $E_{i-1}=E$ if $\left.\Delta t_{i} \in\right] \alpha_{1}, \alpha_{2}$ [. This quantity can be obtained deriving the

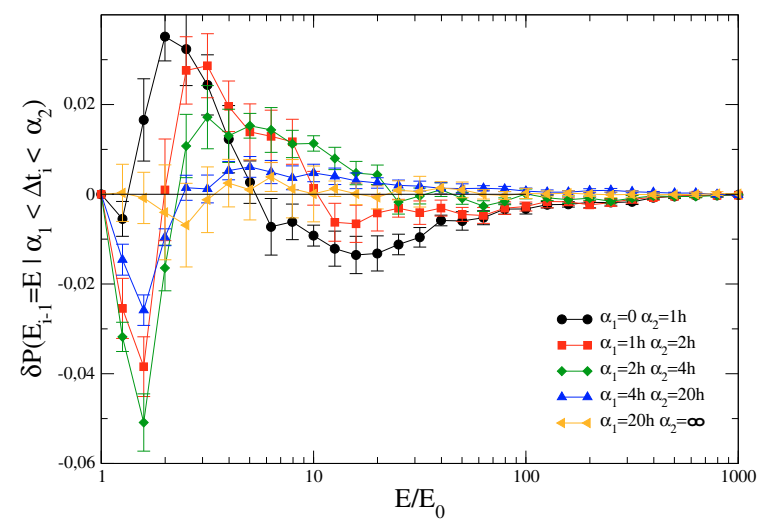

Fig. 5. Probability density difference $\delta p\left(E_{i-1}=E \mid \alpha_{1}<\Delta t_{i}<\alpha_{2}\right)$ versus $E / E_{0}$ for different values of $\alpha_{1}$ and $\alpha_{2}$.

cumulated probability $\delta P\left(E_{i-1}>E \mid \alpha_{1}<\Delta t_{i}<\alpha_{2}\right)$ with respect to $E$. Being a derivative, it is affected by higher fluctuations. For every choice of $\alpha_{1}$, the value of $\alpha_{2}$ was chosen to have the same number of events, about 9000 , for all curves. We noticed that for $\Delta t>\alpha_{1}=20 \mathrm{~h}$ the difference in the probability density $d p$ was very close to zero, indicating the absence of correlations.

We now discuss the results of Fig. 5 at fixed $E / E_{0}$ and for varying $\alpha_{1}$. We first observed that for small of $E, d p$ is always negative for all values of $\alpha_{1}$. The negative region is restricted to very small values of $E \lesssim 1.5 E_{0}$ for $\alpha_{1}=0$ and increases to $E \lesssim 2.3 E_{0}$ for larger $\alpha_{1}$. Within this interval $d p$ is non monotonic in $\alpha_{1}$, in particular, it reaches the minimum value for $\alpha_{1}=2 \mathrm{~h}$ and then tends to zero. For $\Delta t_{i}$ in a given range, negative values of $d p$ imply that it is less probable to have a flare with an energy $E_{i}=E$ compared to the case where $E_{i}$ and $\Delta t_{i}$ are uncorrelated. Results in Fig. 5 then imply that it is improbable to have small $\Delta t$ after very small flares and that these anticorrelations vanish for large $\Delta t$. In particular the highest negative values of $d p$ are obtained for $\Delta t \in[2 \mathrm{~h}, 4 \mathrm{~h}]$ after an $E \lesssim 2 E_{0}$ flare.

If these anticorrelations were a spurious effect of obscuration, we should rather observe a different behavior. Indeed, energy reshuffling produces more events shortly after a large flare compared to the real catalog. Consequently, we should observe an excess, and not a deficit, of events after a small flare in the real catalog compared to the reshuffled one because the number of events is conserved. We now discuss higher values of $E \gtrsim 10 E_{0}$. In this case $d p$ moves from negative values for $\alpha_{1}=0$ and $\alpha_{1}=1 \mathrm{~h}$ to positive values for $\alpha_{1}=2 \mathrm{~h}$ and $\alpha_{1}=4 \mathrm{~h}$ and then tends to zero for higher values of $\alpha_{1}$. This indicates that there is a deficit of events with $\Delta t<2 \mathrm{~h}$ after energetic flares, whereas for $\Delta t \in[2 \mathrm{~h}, 4 \mathrm{~h}]$ the number of observed flares is larger than the one expected for an uncorrelated process. The above results suggest that after a large flare a recovery time of about $2 \mathrm{~h}$ takes place, when only a small number of flares is observed. After this time the number of observed flares reaches a maximum value for $\Delta t \lesssim 4 \mathrm{~h}$ and then decreases to the background level for larger $\Delta t$. According to the dependence on $e_{\text {th }}$ observed in Fig. 4a, the recovery time can be a spurious effect related to obscuration.

The number of events $n(t)$ occurring at the time $t$ after an energetic flare can be explicitly evaluated following the method of Lippiello (2008b). More precisely, we define as a "main" flare an event with the energy $E \geq E_{\text {main }}=10 E_{0}$. Indicating with $t_{i}$ the occurrence times of main-flares we compute the quantity $\mathcal{N}\left(t, E_{\text {main }}\right)=\sum_{i} n\left(t-t_{i}\right) \Theta\left(t_{i+1}-t\right)$, where $\Theta(x)$ is the Heaviside step function, and the sum extends over all main-flares. 

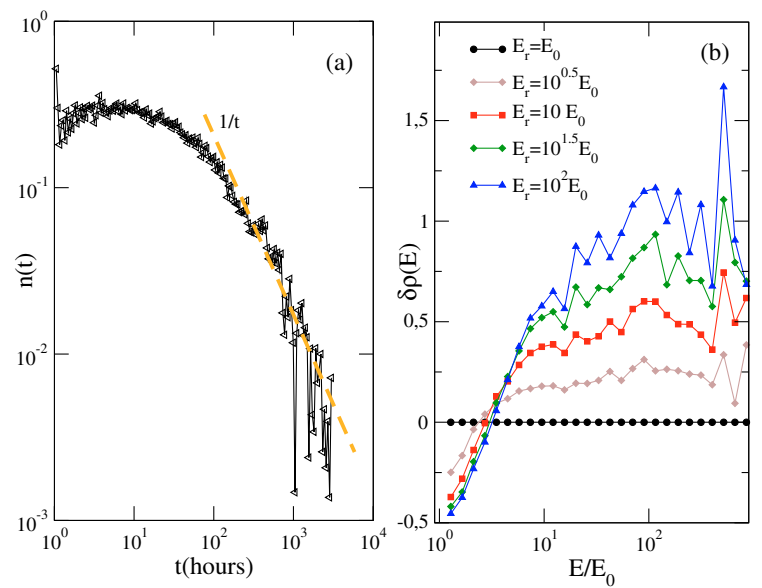

Fig. 6. a) Average number of flares $n(t)$ as a function of the time $t$ occurring after a main flare with an energy $E \geq E_{\text {main }}=10 E_{0}$. The data are averaged over 4057 main flares. b) The difference $\delta \rho(E)$ between the energy distribution for the first $m=20$ flares occurring after an event with a higher energy than $E_{r}$ and the entire catalog energy distribution and different $E_{r}$ values.

Here $n\left(t-t_{i}\right)$ is the number of flares with an energy lower than $10 E_{0}$ occurring during the time $t-t_{i}$ after the $i$ th main flare, and the sum extends over all main-flares in the catalog. Assuming that $n\left(t-t_{i}\right)$ is time translationally invariant we obtain

$n(t)=\frac{\mathcal{N}\left(t, E_{\text {main }}\right)}{\sum_{i} \Theta\left(t_{i+1}-t\right)}$,

which represents the average number of flares occurring in a period of a duration $t$ after a mainflare. The data (Fig. 6a) agree with the previous results, with a maximum number of flares at $t \simeq 4 \mathrm{~h}$, decaying at longer times. Notice that the asymptotic decay is consistent with a power law $t^{-p}$ with $p \simeq 1$, reminiscent of the Omori law for seismic sequences (Omori 1894; de Arcangelis et al. 2006).

We now discuss the existence of correlations between energies of subsequent flares. In order to do so, we computed the energy distribution $\rho(E)$ of the first $m$ flares occurring after a flare with a larger energy than a reference value $E_{r}$. Since the analysis is restricted to all flares with $E \geq E_{0}$, obviously $\rho(E)$ coincides with the flare energy distribution of the whole catalog $\rho_{T}(E)$, for $E_{r}=E_{0}$. In Fig. 6b we plot $\delta \rho(E)=\rho(E)-\rho_{T}(E)$ for $m=20$ and different values of $E_{r}$. Similar results are obtained for other values of $m$. Deviations from $\rho_{T}(E)$ become more and more evident for increasing values of $E_{r}$. In particular, the higher $E_{r}$ is, the larger is the probability to have subsequent flares with higher energy and the lower is the probability to have small flares.

In order to get further insights in the correlations between flare energies, we computed the quantity $\delta p\left(E_{i}=\lambda E_{i-1} \mid \Delta t_{i}<\right.$ $T)$, i.e. the difference between the conditional and the unconditional probability density to have the energy of the subsequent flare $\lambda$ times the previous one. We found (Fig. 7a) that for $T=1 \mathrm{~h}, \delta p\left(E_{i}=\lambda E_{i-1} \mid \Delta t_{i}<T\right)$ is significantly different from zero for all $\lambda$ values. This indicates that the energies of two subsequent flares are correlated. Moreover we observed that these correlations depend on the time separation between the two flares and are practically zero for $\Delta t_{i}>10 \mathrm{~h}$ (Fig. 7a). We verified that this result is not affected by obscuration performing the same analysis for $T=1 \mathrm{~h}$ and different lower energy thresholds $e_{\text {th }}$ (Fig. 7b). We found that curves for different $e_{\text {th }}$ coincide within statistical fluctuations. This indicates that energy correlations and their dependence on time separation are a physical

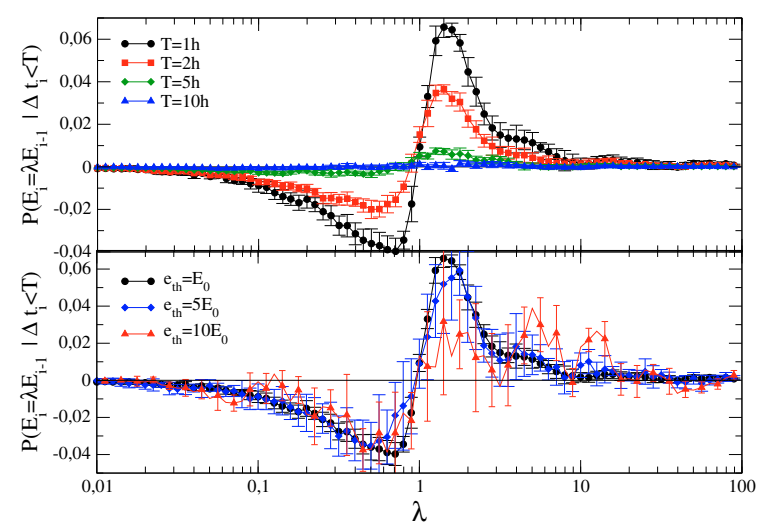

Fig. 7. Probability density difference $\delta p\left(E_{i}=\lambda E_{i-1} \mid \Delta t_{i}<T\right)$ as a function of $\lambda$ for different $T$ a) and for $T=1 \mathrm{~h}$ and different $e_{\mathrm{th}} \mathbf{b}$ ).

property, not a spurious effect due to obscuration. Curves present a maximum for $\lambda \gtrsim 1$, indicating that it is more probable to find the next flare with an energy close to but slightly higher than the previous one.

\section{Conclusions}

In conclusion, we presented a statistical analysis of the GOES catalog indicating the existence of time-energy correlations between successive events not to be attributed to obscuration effects. More precisely, we observed that for couples of events close in time $(T<1 \mathrm{~h})$, the second event tends to have a high energy. Moreover couples of events distant in time between $1 \mathrm{~h}$ and $10 \mathrm{~h}$ have the first event with a high energy. The analysis of the rate decay after large flares shows evidence that the largest number of events is detected about $4 \mathrm{~h}$ after the occurrence of the main event. Finally the distribution of flare energies confirms that the higher the flare energy, the larger the number of subsequent events with high energy. The existence of time-energy correlations suggests the possibility of scaling laws relating time with the energy released in a flare. This is a still open question, which could provide interesting insights in the energy storage and release mechanisms at the origin of solar flare occurrence.

\section{References}

Aschwanden, M. J., Dennis, B. R., \& Benz, A. O. 1998, ApJ, 497, 972 Boffetta, G., Carbone, V., Giuliani, P., Veltri, P., \& Vulpiani, A. 1999, Phys. Rev. Lett., 83, 4662

Crosby, N., Vilmer, N., Lund, N., \& Sunyaev, R. 1998, A\&A, 334, 299 de Arcangelis, L., Godano, C., Lippiello, E., \& Nicodemi, M. 2006, Phys. Rev. Lett., 96, 051102

Hamon, D., Nicodemi, M., \& Jensen, H. J. 2002, A\&A, 387, 326

Jensen, H. J. 1998, Self Organized Criticality (Cambridge University Press)

Lee, T. T., Petrosian, V., \& McTiernan, J. M. 1993, ApJ, 412, 401

Lippiello, E., de Arcangelis, L., \& Godano, C. 2008a, Phys. Rev. Lett., 100, 038501

Lippiello, E., de Arcangelis, L., \& Godano, C. 2008b, A\&A, 488, L29

Lu, E. T., \& Hamilton, R. J. 1991, ApJ, 380, L89

Norman, J. P., Charbonneau, P., McIntosh, S. W., \& Liu, H. 2001, ApJ, 557, 891

Omori, F. 1894, J. Coll. Sci. Imp. Univ. Tokyo, 7, 111

Priest, E. R., \& Forbes, T. G. 2002, A\&ARv, 10, 313

Rosner, R., \& Vaiana, G. S. 1978, ApJ, 222, 1104

Sahraoui, F. 2008, Phys. Rev. E, 78, 026402

Schreiber, T., \& Schmitz, A. 2000, Physica D, 142, 346

Theiler, J., Eubank, S., Longtin, A., Galdrikian, B., \& Doyne Farmer, J. 1992, Physica D, 58, 77

Wheatland, M. S. 2000, Sol. Phys., 191, 381

Wheatland, M. S., \& Litvinenko, Y. E. 2002, Sol. Phys., 211, 255

Wheatland, M. S., Sturrock, P. A., \& McTiernan, J. M. 1998, ApJ, 509, 448 